\title{
Two New Species of Quadrisegmentum (Phtisicidae: Amphipoda: Crustacea) from the Central Indo-Pacific, with Notes on the Type Species $Q$. triangulum Hirayama, 1988
}

\author{
LAUREN ElizABETH HUGHES ${ }^{1 *}$ AND ICHIRO TAKEUCHI ${ }^{2}$ \\ ${ }^{1}$ Australian Museum Research Institute, \\ Australian Museum, 1 William Street, Sydney New South Wales 2010, Australia \\ ${ }^{2}$ Department of Life Environment Conservation, and Center of Advanced Technology for the Environment, \\ Faculty of Agriculture, Ehime University, 3-5-7 Tarumi, Matsuyama, Ehime 790-8566, Japan \\ lauren.hughes@austmus.gov.au ·takeuchi@agr.ehime-u.ac.jp
}

\begin{abstract}
Two new species of Quadrisegmentum (Phtisicidae: Amphipoda: Crustacea) are described from the Central Indo-Pacific. The new species Quadrisegmentum atauro sp. nov., is described from the Wetar Basin, Timor-Leste in the Lesser Sunda Region of the Western Coral Triangle. The new species name Q. yirrgay sp. nov., is established for material from Ashmore Reef in Queensland and Papua New Guinea originally reported under the name Q. triangulum Hirayama, 1998 (Guerra-García 2003, 2006; Guerra-García \& Lowry 2009). Study of the type material of Quadrisegmentum triangulum described from Ashmore Reef in the Timor Sea, Western Australia confirms the absence of projections on pereonite 2 , which prompted reassessment of subsequent identifications. Records of $Q$. triangulum are now limited to the type locality only. An updated key to the four known species of Quadrisegmentum is provided.
\end{abstract}

KEYWORDS: Quadrisegmentum; new species; Phtisicidae; Amphipoda; Crustacea; taxonomy

Hughes, Lauren ElizABeth, AND ICHIRO TAKeuChI. 2016. Two new species of Quadrisegmentum (Phtisicidae: Amphipoda: Crustacea) from the Central Indo-Pacific, with notes on the type species Q. triangulum Hirayama, 1988. Records of the Australian Museum 68(5): 231-244. http://dx.doi.org/10.3853/j.2201-4349.68.2016.1663

Much work remains to be done on the marine amphipod fauna of the Central Indo-Pacific. In the Western Coral Triangle where the species diversity of coral is the highest in the world (Veron et al., 2009), 28 caprellid species from 21 genera have been reported from shallow-waters in the Indonesian Archipelago (Mayer, 1903; McCain \& Steinberg, 1970; Krapp-Schickel \& Guerra-García, 2005; Scinto et al., 2008). In the Eastern Coral Triangle and Northeast
Australian Shelf (Spalding et al., 2007), three previous studies documented 19 species and 15 genera from these two regions combine (Guerra-García, 2003, 2006; Lowry \& Guerra-García, 2009).

The genus Quadrisegmentum was established by Hirayama, 1988 to account for one species, Q. triangulum, collected from Ashmore Reef in the Timor Sea. Research by Guerra-García (2006) described a second species of

* author for correspondence 
Quadrisegmentum, Q. lowryi Guerra-García, 2006, from Lizard Island on the northern Great Barrier Reef. GuerraGarcía $(2003,2006)$ further reported $Q$. triangulum from the Lord Howe Island, Elizabeth and Middleton Reef, the Great Barrier Reef, Ashmore Reef (Coral Sea) and Papua New Guinea.

The present study contributes an additional new species and generic record, Quadrisegmentum atauro, from TimorLeste, which is part of the Lesser Sunda region within the Western Coral Triangle. Quadrisegmentum atauro has distinct projections of pereonite 2. Comparative examination of $Q$. triangulum type material confirmed the absence of projections on pereonite 2 , yet in material subsequently attributed to Q. triangulum (see Guerra-García, 2003, 2006), pereonite 2 projections were observed on male and female species from various locations. Following reassessment of the latter material, a second new species $Q$. yirrgay, is established for previous records of $Q$. triangulum reported throughout the Coral Sea and Bismark Archipelago (Eastern Coral Triangle and Northeast Australian Shelf, respectively).

\section{Materials and methods}

The material examined for this study included type material from the Museum and Art Gallery of the Northern Territory (NTM), Australian Museum (AM) collections of Quadrisegmentum and new collections made during the Australian Museum Timor-Leste Expedition in September 2012 (Hughes, 2015). Material was dissected in $80 \%$ ethanol. Temporary slides were made using $100 \%$ glycerol. Permanent slides were made using either Polyvinyl lactophenol or Aquatex ${ }^{\mathrm{TM}}$ (Merck, Darmstadt, Germany) mounting agent. Illustrations were made with a Laborlux K (Leitz Wetzlar, Germany) and Heerbrugg stereomicroscopes (Wilde, Switzerland) fitted with camera lucida. Specimens were prepared for scanning electron microscopy as follows: placed in an ultrasonic cleaning bath for 10 seconds in a $10 \%$ solution of Tween 80 surfactant; preserving solution was returned to $80 \%$ ethanol then sequentially advanced in 5\% increments from $80 \%$ to $100 \%$ ethanol; critical point dried; mounted individually on pins and gold sputter coated. Images were captured using on a Zeiss EVO LS15 Scanning Electron Microscope with Robinson Backscatter Detector (SEM) (Zeiss, Germany). Abbreviations for parts are as follows: $A$, antenna; $G$, gnathopod; $P$, pereopod and $U r$, urosome. Family classification follows Takeuchi (1993).

\section{Systematics section}

\section{Phtisicidae Vassilenko, 1968}

\section{Quadrisegmentum Hirayama, 1988}

Diagnosis. Head fused (suture absent) with pereonite 1 . Antenna 1 well developed; flagellum with more than 2 articles. Antenna 2 well developed; flagellum with more than 2 articles. Mandible well developed; molar absent; palp 3-articulate; article 3 with 1 distal and 1 (or 2) lateral setae. Maxilliped well developed; inner plate (basal endite) larger than outer plate (ischial endite); outer plate (ischial endite) well developed; palp article 3 with distal projection; palp article 4 well developed. Pereonite 4 clavate appendage absent. Pereonites 6 and 7 separated. Pereopod 3 well developed, with 7 articles. Pereopod 4 well developed, with 7 articles. Pereopod 5, with 4 articles, and well-developed dactylus. Pereopods 6 and 7 well developed, with 7 articles. Gills on pereonites 2 to 4 . Uropods 2 pairs; Uropod 1 biarticulate, uniramous. Uropod 2 biarticulate, uniramous. Telson dorsal lobe present.

Included species: Quadrisegmentum triangulum Hirayama, 1988, type species; Q. atauro sp. nov.; Q. lowryi GuerraGarcía, 2006; Q. yirrgay sp. nov.

Remarks. Characters which define the family Phtisicidae Vassilenko, 1968 are a fully fused head and pereonite 1, absence of a mandibular molar and 3-articulate palp, gills on pereonites 2-4 (3 pair), pereonites 5 and 6 separated and the urosomites 1 and 2 coalesced (see Takeuchi, 1993, 2015; Lim et al., 2012; Takeuchi and Lowry, 2016). The diagnosis of the genus was based observations of the type material of $Q$. triangulum Hirayama, 1988 by the present study with reference to description and figures of mouth parts in Hirayama (1988) and additionally the two new species described in the current study, $Q$. atauro sp. nov. and $Q$. yirrgay sp. nov. (map Fig. 11) provides the distribution of the four known Quadrisegmentum species.

\section{Quadrisegmentum atauro sp. nov.}

$$
\text { Figs } 1-4
$$

Type material. Holotype ${ }^{\lambda}, 10.5 \mathrm{~mm}$ (illustrated), dissected in part, 1 slide, AM P.98327, Timor-Leste, east of Atauro Island, Outer Reef, dense coral reef slope ( $\left.8^{\circ} 13^{\prime} 48^{\prime \prime} \mathrm{S} 125^{\circ} 36^{\prime} 57^{\prime \prime E}\right), 17 \mathrm{~m}$, green calcareous alga Halimeda sp., 20 September 2012, coll. L.E. Hughes (TM 2012-087); paratype +, $9 \mathrm{~mm}$ (illustrated), AM P.98328, Timor-Leste, east of Atauro Island, Outer Reef, dense coral reef slope ( $\left.8^{\circ} 13^{\prime} 48^{\prime \prime} \mathrm{S} 125^{\circ} 36^{\prime} 57^{\prime \prime E}\right), 17 \mathrm{~m}$, green calcareous alga Halimeda sp., 20 September 2012, coll. L.E. Hughes (TM 2012-087). Paratypes: 1, $5.1 \mathrm{~mm}$ [a smaller specimen has been chosen for SEM as it is in better condition], whole animal SEM pin mount, AM P.91095, Timor-Leste, east of Atauro Island, Outer Reef, dense coral reef slope ( $\left.8^{\circ} 13^{\prime} 48^{\prime \prime S} 125^{\circ} 36^{\prime} 57^{\prime \prime E}\right), 17 \mathrm{~m}$, green calcareous alga Halimeda $\mathrm{sp}$., 20 September 2012, coll. L.E. Hughes (TM 2012-087); 1 đ̂, 10.5 mm, SEM pin mount, AM P.91091, Timor-Leste, east of Atauro Island, Outer Reef, dense coral reef slope $\left(8^{\circ} 13^{\prime} 48^{\prime \prime} \mathrm{S} 125^{\circ} 36^{\prime} 57^{\prime \prime} \mathrm{E}\right), 17 \mathrm{~m}$, green calcareous alga Halimeda sp., 20 September 2012, coll. L.E. Hughes (TM 2012-087); 3 A . AM P.91097, Timor-Leste, east of Atauro Island, Outer Reef, dense coral reef slope $\left(8^{\circ} 13^{\prime} 48^{\prime \prime S} 125^{\circ} 36^{\prime} 57^{\prime \prime E}\right), 17 \mathrm{~m}$, green calcareous alga Halimeda sp., 20 September 2012, coll. L.E. Hughes (TM 2012-087); 1 જ, AM P.91092, Timor-Leste, east of Atauro Island, Outer Reef, dense coral reef slope ( $\left.8^{\circ} 13^{\prime} 48^{\prime \prime} \mathrm{S} 125^{\circ} 36^{\prime} 57^{\prime \prime} \mathrm{E}\right), 17 \mathrm{~m}$, green calcareous alga Halimeda sp., 20 September 2012, coll. L.E. Hughes (TM 2012-087); 1 mature + , AM P.91096, Timor-Leste, east of Atauro Island, Outer Reef, dense coral reef slope ( $\left.8^{\circ} 13^{\prime} 48^{\prime \prime} \mathrm{S} 125^{\circ} 36^{\prime} 57^{\prime \prime} \mathrm{E}\right), 17 \mathrm{~m}$, green calcareous alga Halimeda sp., 20 September 2012, coll. L.E. Hughes (TM 2012-087); many specimens, AM P.91094, Timor-Leste, east of Atauro Island, Outer Reef, dense coral reef slope ( $\left.8^{\circ} 13^{\prime} 48^{\prime \prime} \mathrm{S} 125^{\circ} 36^{\prime} 57^{\prime \prime E}\right), 17 \mathrm{~m}$, green calcareous alga Halimeda sp., 20 September 2012, coll. L.E. Hughes (TM 2012-087); 4 mature 우우, AM P.91093, Timor-Leste, east of Atauro Island, Outer Reef, dense coral reef slope ( $\left.8^{\circ} 13^{\prime} 48^{\prime \prime} \mathrm{S} 125^{\circ} 36^{\prime} 57^{\prime \prime} \mathrm{E}\right), 17 \mathrm{~m}$, green calcareous alga Halimeda sp., 20 September 2012, coll. L.E. Hughes (TM 2012-087); 7 specimens ( $6 \hat{\delta} \hat{o}$ and 1 mature + ), AM P.91090, Timor-Leste, east of Atauro Island, Inner Reef, reef slope ( $\left.8^{\circ} 14^{\prime} 30^{\prime \prime S} 125^{\circ} 36^{\prime} 49^{\prime \prime} \mathrm{E}\right), 14 \mathrm{~m}$, mixed turfing algae, 20 September 2012, coll. L.E. Hughes (TM 2012-034); 1 mature 9 , AM P.91099, Timor-Leste, west of Dili, off Tibar, on outer side of reef, rubble and sandy reef slope $\left(8^{\circ} 33^{\prime} 48^{\prime \prime} \mathrm{S} 125^{\circ} 28^{\prime} 31^{\prime \prime E}\right), 10-12 \mathrm{~m}$, coral rubble and mixed algae, 21 September 2012, coll. A. Murray (TM 2012-037); 9 specimens (5 $\bar{\delta} \hat{\sigma}$ and 4 mature + 우), AM P.91098, Timor-Leste, east of Metinaro, Secret Garden Reef ( $\left.8^{\circ} 29^{\prime} 15^{\prime \prime S} 125^{\circ} 49^{\prime} 53^{\prime \prime E}\right), 20 \mathrm{~m}$, tufts of red algae, 22 September 2012, coll. L.E. Hughes (TM 2012-060). 

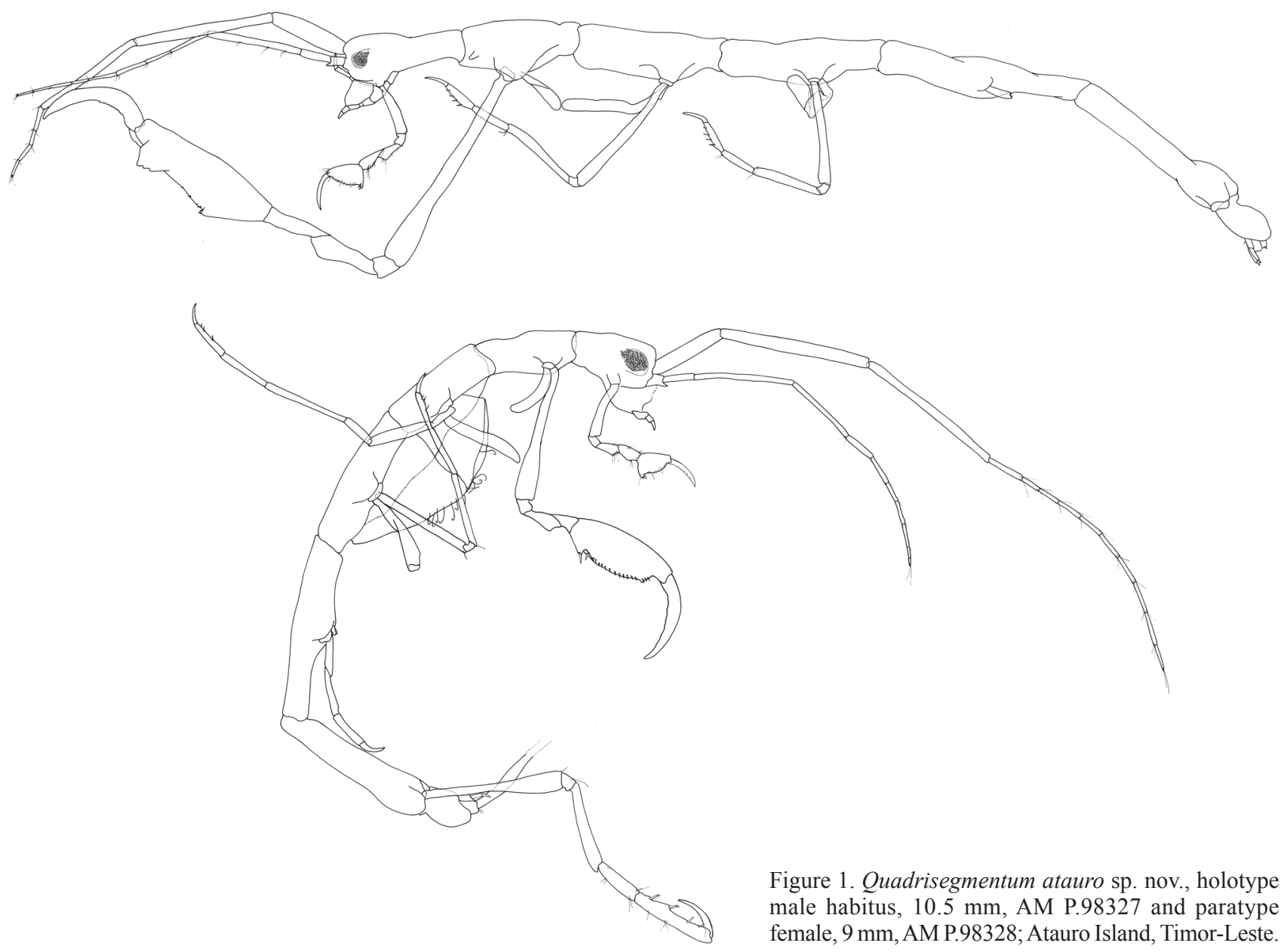

Figure 1. Quadrisegmentum atauro sp. nov., holotype male habitus, $10.5 \mathrm{~mm}$, AM P.98327 and paratype female, 9 mm, AM P.98328; Atauro Island, Timor-Leste.
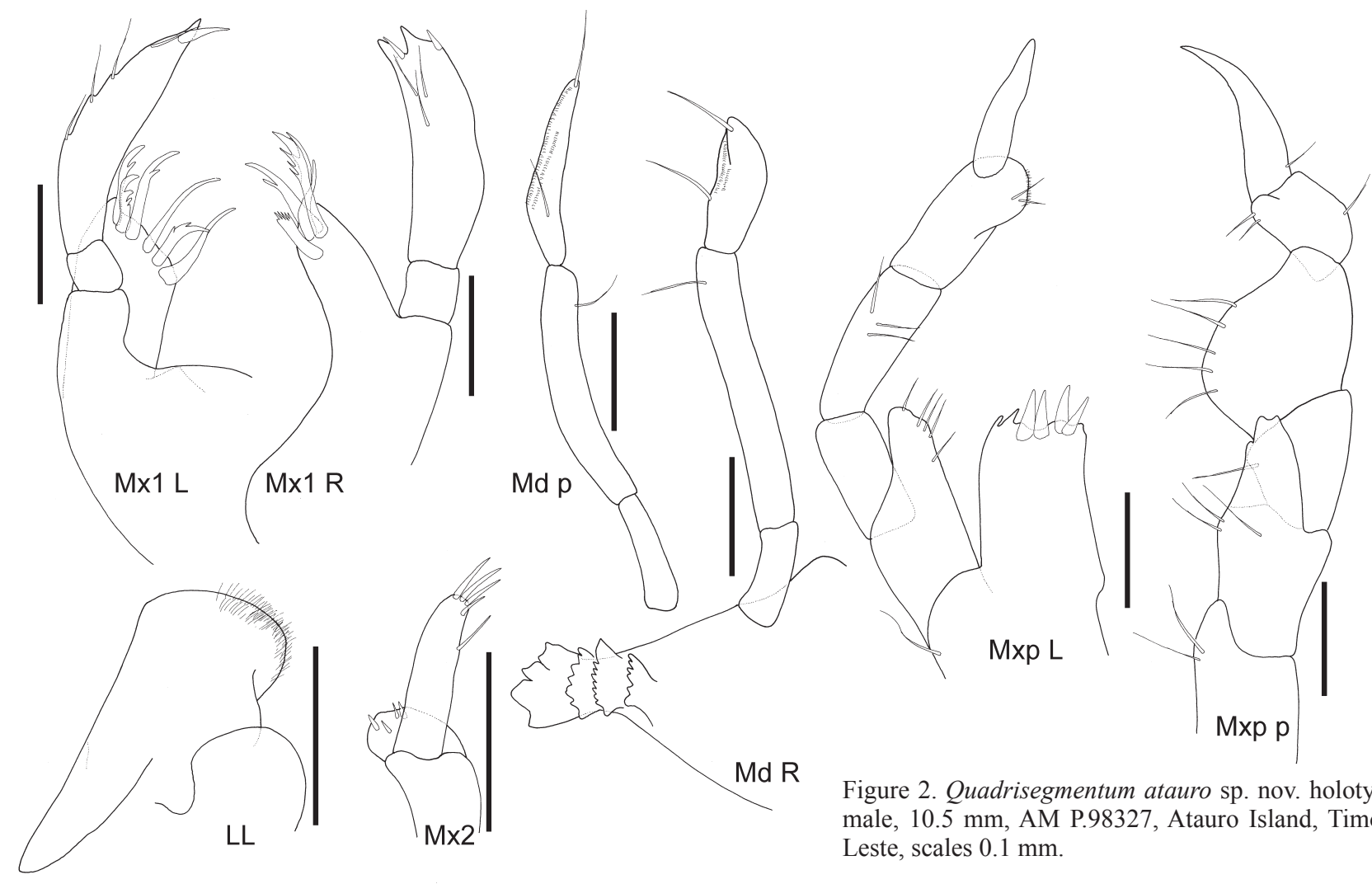

Figure 2. Quadrisegmentum atauro sp. nov. holotype male, 10.5 mm, AM P.98327, Atauro Island, TimorLeste, scales $0.1 \mathrm{~mm}$. 


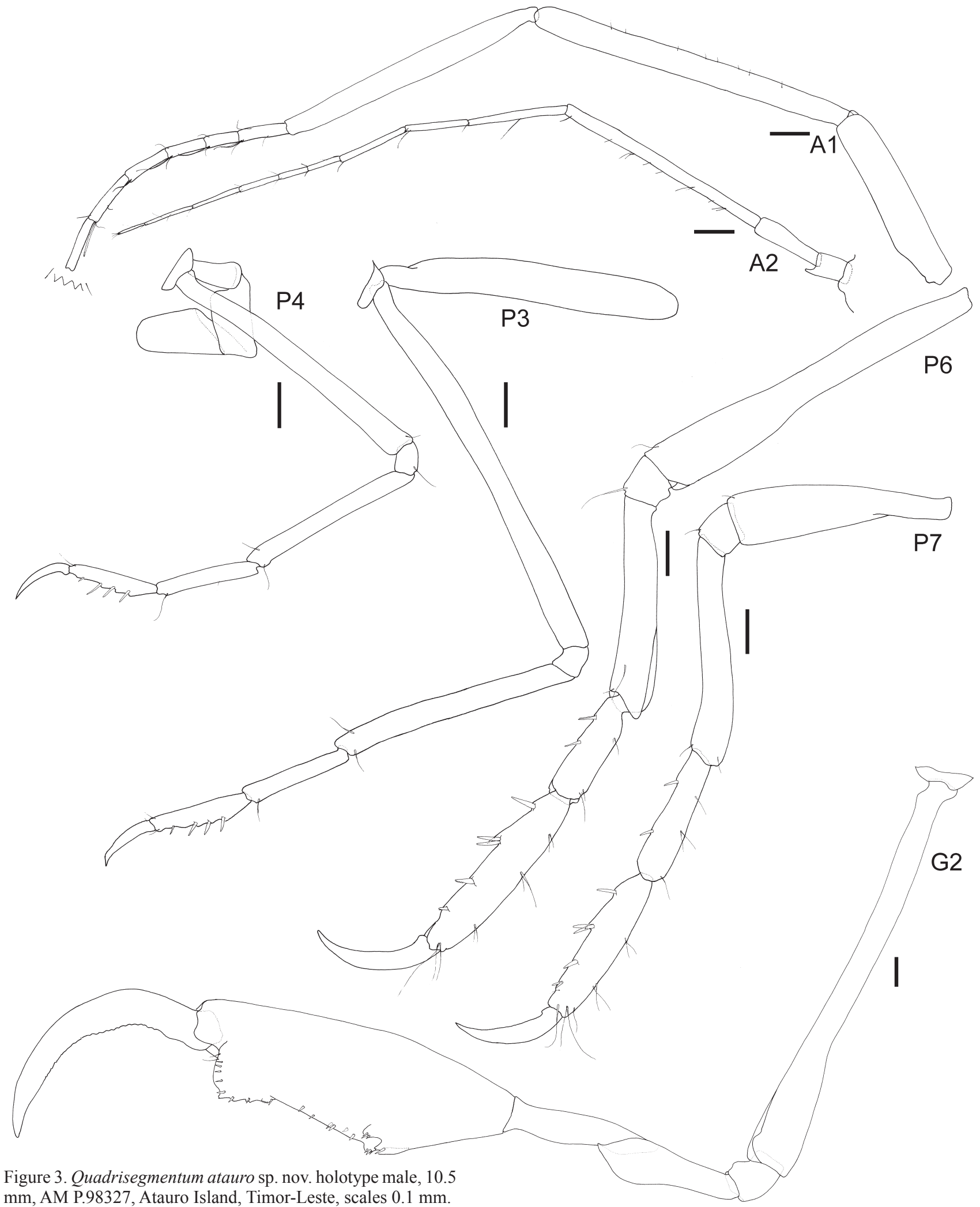
mm, AM P.98327, Atauro Island, Timor-Leste, scales $0.1 \mathrm{~mm}$.

Type locality. Timor-Leste, east of Atauro Island, Outer Reef ( $\left.8^{\circ} 13^{\prime} 48^{\prime \prime} \mathrm{S} 125^{\circ} 36^{\prime} 57^{\prime \prime} \mathrm{E}\right)$.

Etymology. Named from the type locality, applied as a noun in apposition.

Description. (Based on male, body length, $10.5 \mathrm{~mm}, \mathrm{AM}$ P. 98327, and male, body length, 10.5 mm, AM P.91901 for pereopod 5 and abdomen).
Body Somites slender. Head and pereonite 1 combined, completely fused, suture absent. Pereonite 2 with small acute anterolateral projection. Pereonites 3-6 without anterior or mid-lateral projections.

Antenna $11 / 3 \times$ body length; peduncle article 2 longest, $1.9 \times$ article 1 ; article 3 length $0.8 \times$ article 2 ; flagellum length $0.24 \times$ peduncle with $4-6$ articles (left and right, respectively). Antenna $20.9 \times$ Antenna 1 ; flagellum, $0.25 \times$ peduncle length, body with 8-9 articles (left and right, respectively). 


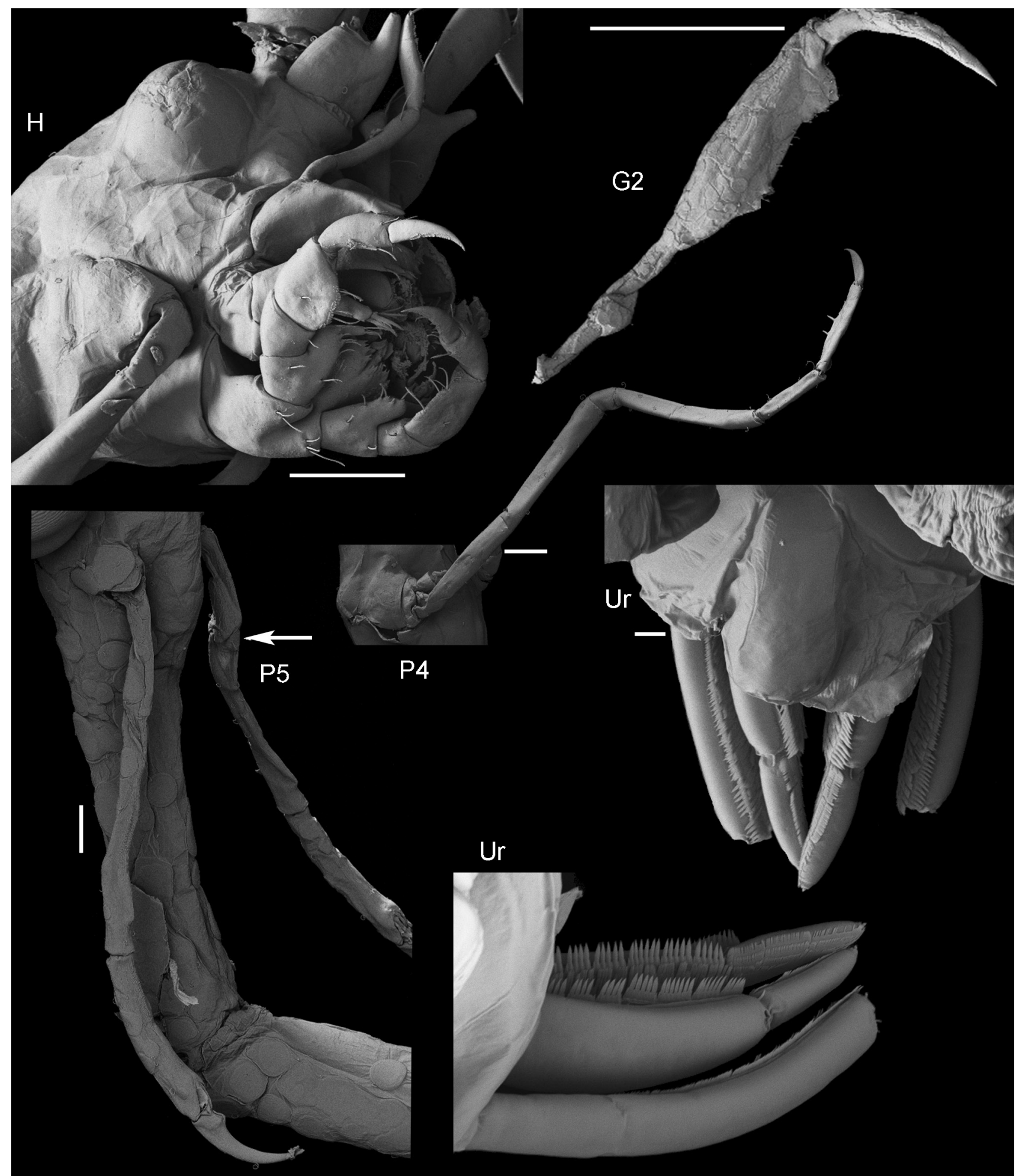

Figure 4. Quadrisegmentum atauro sp. nov. SEM image of paratype male, $5.1 \mathrm{~mm}$, head and pereopod 4, AM P.91095; paratype male, $10.5 \mathrm{~mm}$, gnathopod 2, pereopod 5 and urosome paratype male AM P.91091; Atauro Island, Timor-Leste. Scales: H and P4-5 scale 100 $\mu \mathrm{m}$, Ur dorsal view scale $10 \mathrm{~mm}$ and $\mathrm{Ur}$ transverse view $20 \mu \mathrm{m}$.

Mandible right similar to left, incisor with 4 teeth, lacinia mobilis with 7 teeth followed by 2 plates; palp 3-articulate; article 2 longest, with 1 distal slender setae; article 3 with 1 distal and 1 midlateral slender setae, anterodistal margin pubescent. Mandible left, palp similar to right palp. Lower lip setose on outer lobe. Maxilla 1 outer plate with 5-6 robust setae; palp 2-articulate, article 2 with 2 triangular projections, each with a robust seta, with 3-4 subapical slender setae. Maxilla 2 inner plate short with 4 robust setae; outer plate long, with 4 apical and 1 subapical robust setae. Maxilliped basal endite (inner plate) larger than ischial endite (outer plate), rectangular, with 4 apically robust setae near distal margin; ischial endite (outer plate) several slender setae on inner margin; palp 4-articulate; article 2 laterally expanded, with 3-4 slender setae; article 3 with rounded distal projection with 2 setae. 
Pereon. Gnathopod 1 basis similar in length to ischium, merus and carpus combined; carpus and merus subequal in length with anterior and posterior slender setae; propodus triangular; palm defined by prominent, curved, bifid process with 2 apical robust setae; dactylus falcate, inner margin lined with setules. Gill 2 elongate, length $5 \times$ width, $0.6 \times$ pereonite 2 length. Gnathopod 2 begins $1 / 3$ along posterior margin of pereonite 2 ; basis length $1.9 \times$ pereonite 2 length, 1.6× ischium, merus and carpus combined; merus subrectangular, distally swollen (length $2.8 \times$ width), subequal to carpus; carpus subrectangular (length $3.1 \times$ width); propodus subovate, longer than wide (length $2.8 \times$ width); palm $60 \%$ of posterior margin, 3 proximal projections each with robust seta.

Gill 3 slender, $7 \times$ width, length $0.9 \times$ pereonite 3 length. Pereopod 3 length $0.3 \times$ body length; basis longest, $0.4 \times$ pereopod 3 length; ischium $0.1 \times$ basis length; merus $0.6 \times$ basis length; carpus $0.27 \times$ basis length; propodus $0.9 \times$ carpus length with 3 large and 1 small robust setae along palm; dactylus recurved. Gill 4 subequal to gill 3 . Pereopod 4 shorter than pereopod 3, length $0.35 \times$ body length; basis longest, $0.4 \times$ pereopod length; ischium $0.06 \times$ basis length; merus $0.65 \times$ basis length; carpus $0.3 \times$ basis length; propodus shorter than carpus, length $0.9 \times$ carpus with 3 large and 1 small robust setae along palm; dactylus recurved.

Pereopod 5 with 4 articles; article 1 length $0.9 \times$ article 2; article 2 longest, $1.1 \times$ article 3 ; dactylus recurved. Pereopod 6 slender, $0.5 \times$ body length; basis longest, $0.37 \times$ pereopod 6 length; ischium $0.08 \times$ basis length; merus $0.7 \times$ basis length; carpus $0.33 \times$ basis length, anterior margin with 2 robust setae; propodus $0.6 \times$ basis length, anterior margin with 3 groups of long robust setae (middle group only with paired robust setae) and distal small robust setae; dactylus recurved. Pereopod 7 slender, smaller than pereopod 6, 0.4× body length; basis $0.27 \times$ pereopod 7 length; ischium $0.25 \times$ basis length; merus length subequal to basis; carpus $0.65 \times$ basis length, anterior margin with 2 robust setae; propodus $0.8 \times$ basis length, anterior margin with 3 groups of long robust setae (middle group only with paired robust setae) and distal small robust setae; dactylus recurved.

Pleon. Uropod 1 elongate (length $6 \times$ width), with minute a line of lateral setae; ramus damaged. Uropod 2 peduncle elongate (length $2.5 \times$ width), with a line of lateral setae; ramus $0.6 \times$ peduncle length. Telson small, with a pair of setae.

Female. (based on female paratype, body length $9 \mathrm{~mm}$, AM P. 98328). Pereonite 4 without rounded anterolateral projection. Antenna 1 article 2 subequal to article 3 length, $1.8 \times$ article 1 length; flagellum with 10 articles. Antenna 2 near $2 / 3$ length of antenna 1 ; flagellum $0.6 \times$ peduncle length with 7 articles.

Remarks. Quadrisegmentum atauro sp. nov. and Q. yirrgay sp. nov. have long rectilinear gills which separates them from $Q$. triangulum where the gills are much shorter and subovate. The pereonite 2 in Q. atauro sp. nov. has on one pair of acute anterior lateral projections which are not seen for Q. yirrgay sp. nov. and Q. lowryi Guerra-García, 2006.

Distribution. Timor-Leste: Atauro Island, Tibar (current study).

\section{Quadrisegmentum triangulum Hirayama, 1988}

Fig. 5

Quadrisegmentum triangulum —Hirayama, 1988: 10901093, figs. 1-3.

not Quadrisegmentum triangulum. - Guerra-Garcia, 2003: 104-105, fig. 9a. -Guerra-Garcia, 2006: 440, fig. 39.

—Guerra-Garcia \& Lowry, 2009: 323-325 (= Q. yirrgay sp. nov.).

Type material examined. Paratypes $1 \hat{\delta}$ (no. 1) $8 \mathrm{~mm}$, and 2 우 (nos. 2-3) $5.2 \mathrm{~mm}$ and unmeasured, NTM Cr. 00447, taken from gorgonian host, Isis hippuris Linnaeus, 1758 in $18 \mathrm{~m}$ on west islet of Ashmore Reef, Northwest Australia (12 $14.28^{\prime} \mathrm{S} 122^{\circ} 59.14^{\prime} \mathrm{E}$.), 24 July 1986 , coll. H. K. Larson. (Holotype carcass and slide material not found, presumed lost).

Type locality. Ashmore Reef, Timor Sea, north-west Western Australia $\left(12^{\circ} 14.28^{\prime} \mathrm{S} 122^{\circ} 59.14^{\prime} \mathrm{E}\right)$.

Remarks. Examination of Q. triangulum type material recognizes the absence of projections on pereonite 2 in both sexes and also records the presence of anterolateral projections on the male pereonite 3 . These anterolateral projections on pereonite 3 were not recorded in the original description by Hirayama (1988). Additional material attributed to $Q$. triangulum by the first and second reviewer from Queensland, Australian and Papua New Guinea (Guerra-García, 2003, 2006), contained specimens where pereonite 2 projections were observed on males and females. This latter material was reassessed as part of this study and determined to be a separate species. These records are transferred to the new species name $Q$. yirrgay described below. The name Quadrisegmentum triangulum is once again restricted to the original type material and presently known only from the type locality.

Distribution. Ashmore Reef (Hirayama, 1988).

\section{Quadrisegmentum yirrgay sp. nov.}

Figs 6-10

Quadrisegmentum triangulum. - Guerra-Garcia, 2003: 104-105, fig. 9a. -Guerra-Garcia, 2006: 440, fig. 39. —Guerra-Garcia \& Lowry, 2009: 323-325.

Type material. Holotype $\widehat{\delta} 9.0 \mathrm{~mm}$, AM P.98563, outer reef slope, Ashmore Reef, Coral Sea, Queensland, Australia (10²6'16"S 144²5'47"E), red calcareous cylindrical algae, 19 January 1993, coll. S.J. Keable (QLD 704); paratype $\$ 9.0 \mathrm{~mm}$, AM P.98564, outer reef slope, Ashmore Reef, Coral Sea, Queensland, Australia $\left(10^{\circ} 26^{\prime} 16^{\prime \prime S} 144^{\circ} 25^{\prime} 47^{\prime \prime E}\right)$, red calcareous cylindrical algae, 19 January 1993, coll. S.J. Keable (QLD 704); many specimens, AM P.61630, outer reef slope, Ashmore Reef, Coral Sea, Queensland, Australia $\left(10^{\circ} 26^{\prime} 16^{\prime \prime S} 144^{\circ} 25^{\prime} 47^{\prime \prime E}\right)$, red calcareous cylindrical algae, 19 January 1993, coll. S.J. Keable (QLD 704). Paratypes: 9, 6 mm, AM P.61624, eastern edge of outer reef slope, Ashmore Reef, Coral Sea, Queensland, Australia $\left(10^{\circ} 09^{\prime} 10^{\prime \prime S} 144^{\circ} 34^{\prime} 43^{\prime \prime E}\right)$ calcareous red tubular algae, 24 January 1993 , coll. S.J. Keable (QLD 734); 2 specimens (1 1 and 1 P), AM P.61627, eastern edge of outer reef slope Ashmore Reef, Coral Sea, Queensland, Australia $\left(10^{\circ} 09^{\prime} 10^{\prime \prime} \mathrm{S} 144^{\circ} 34^{\prime} 43^{\prime \prime E}\right)$ mixed sponges, 24 January 1993, coll. S.J. Keable (QLD 736); 4 specimens (2 30 and 2 우 $)$, AM P.61629, outer reef slope, Ashmore Reef, Coral Sea, Queensland, Australia (10¹3'10"S $144^{\circ} 24^{\prime} 53$ "E) sponges, 22 January 1993, coll. S.J. Keable (QLD 722); 1 ô, AM P.61625, outer reef slope, Ashmore Reef, Coral Sea, Queensland, Australia $\left(10^{\circ} 26^{\prime} 16^{\prime \prime S} 144^{\circ} 25^{\prime} 47^{\prime \prime E}\right)$ sediment, algae and rubble, 19 January 1993, coll S.J. Keable (QLD 705). 


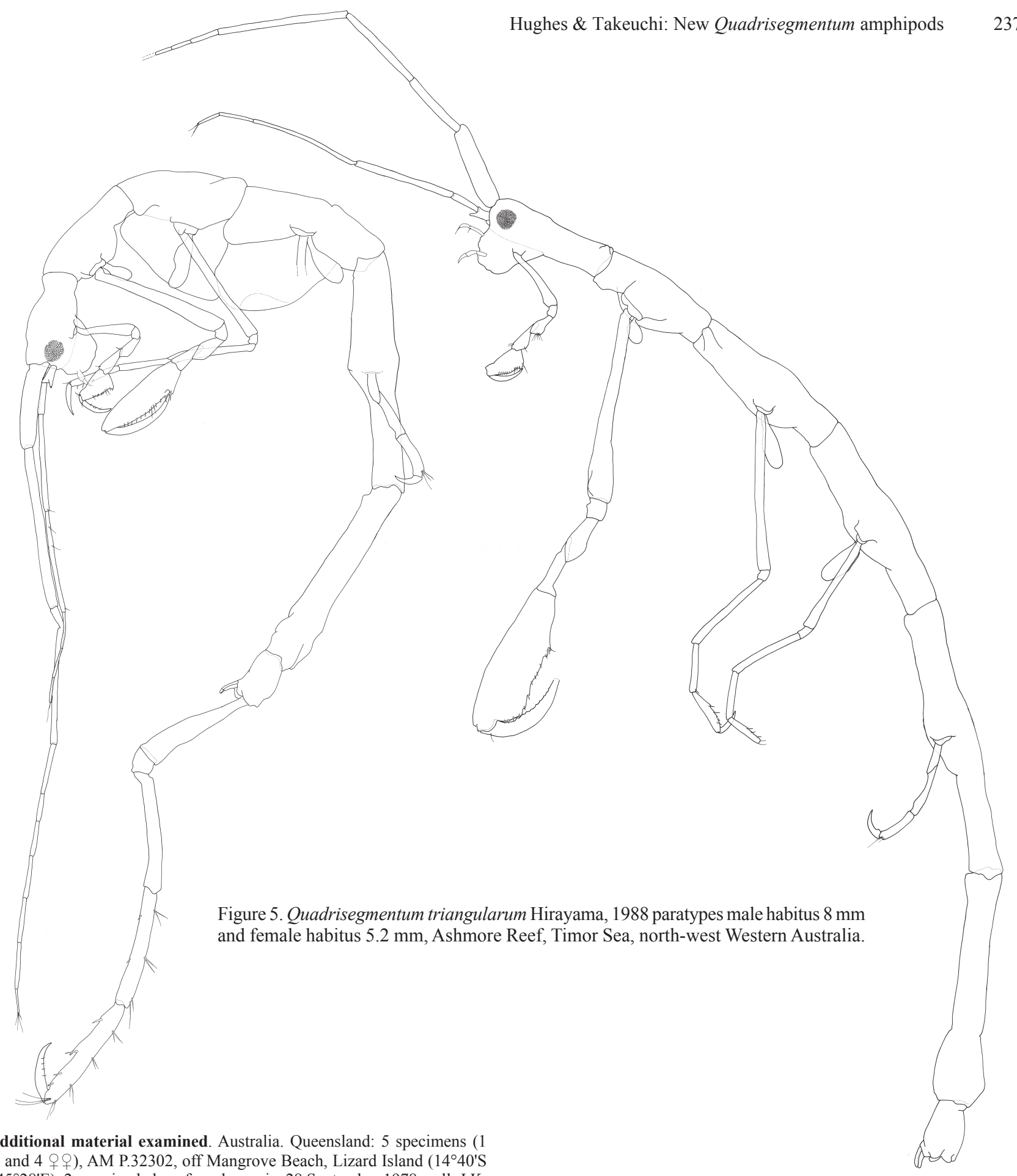
$\pi$ and 4 O ${ }^{\circ}$ ), AM P 32302 off Mangrove Beach, Lizard Island ( $14^{\circ} 40^{\prime} \mathrm{S}$ $\left.145^{\circ} 28^{\prime} \mathrm{E}\right), 2 \mathrm{~m}$, mixed algae from bommie, 28 September 1978, coll. J.K. Lowry (QLD 1); 2 specimens (1 $\delta^{\lambda}$ and 1 P), AM P.32314, off southern Point of Mermaid Cove, Lizard Island (14'39'S $\left.145^{\circ} 27^{\prime} \mathrm{E}\right), 3-6.1 \mathrm{~m}$, filamentous algae with stone washings and scrapings from crevices, 8 October 1978 , coll. C. J. Short \& P.C. Terrill (QLD-25); 1 9, AM P.32318, fringing reef between Bird Islet and South Island, Lizard Island $\left(14^{\circ} 40^{\prime} \mathrm{S} 145^{\circ} 28^{\prime} \mathrm{E}\right)$, $25 \mathrm{~m}$, green algae Halophila sp. and mixed algae from grass beds off reef base, 9 October 1978, coll. J.K. Lowry (QLD 29); 2 đ reefs at western end of Blue Lagoon, Lizard Island (14 $\left.40^{\circ} \mathrm{S} 145^{\circ} 28^{\prime} \mathrm{E}\right), 3$ $\mathrm{m}$, sand near artificial reefs, 5 October 1978, coll. P.C. Terrill (QLD 33); 16 specimens ( 6 ô 0 , 9 우, 1 juvenile), AM P.32325, Mermaid Cove, Lizard Island $\left(14^{\circ} 40^{\prime} \mathrm{S} 145^{\circ} 28^{\prime} \mathrm{E}\right)$, subtidal to $2 \mathrm{~m}$, mixed algae, coral rubble and silt, 14 October 1978, coll. J.K. Lowry (QLD-48); 1 \%, AM P.32326, off North Point, Lizard Island (14³9'S $\left.145^{\circ} 27^{\prime} \mathrm{E}\right), 3-6.1 \mathrm{~m}$, red algae (Galaxaura sp.) and coral rubble from subtidal caves, 14 October 1978, coll. J.K. Lowry (QLD 49); 9 specimens (7 $\widehat{\delta}, 1$, 1 juvenile), AM P.32327, off North Point, Lizard Island (14²39'S $\left.145^{\circ} 27^{\prime} \mathrm{E}\right), 3-6.1 \mathrm{~m}$, mixed algae, 14 October 1978, coll. J.K. Lowry (QLD 50); 7 specimens (4 $\widehat{\delta} \hat{\delta}$ and 3 웅), AM P.32328, Lizard Island, off North Point, Lizard Island (14'39'S $\left.145^{\circ} 27^{\prime} \mathrm{E}\right), 6.1 \mathrm{~m}$, green calcareous alga Halimeda sp., 14

October 1978, coll. P.C. Terrill (QLD 52); 1 त, AM P.61626, Great Barrier

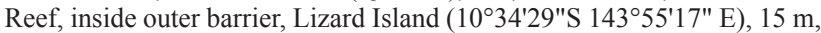
rubble, 16 January 1993, coll. S.J. Keable (QLD 682); 1 đે, AM P.61712, between Palfrey Island and Lizard Island, western side of Blue Lagoon, Lizard Island $\left(14^{\circ} 41^{\prime} \mathrm{S} 145^{\circ} 27^{\prime} \mathrm{E}\right), 7$ October $2001 ; 5$ specimens $\left(2 \delta^{\circ} \delta^{\circ}\right.$ and 3 오), AM P.61713, between Palfrey Island and Lizard Island, western side of Blue Lagoon, Lizard Island (14 $\left.41^{\prime} \mathrm{S} 145^{\circ} 27^{\prime} \mathrm{E}\right), 7$ October 2001; 1 , AM P.61714, between Palfrey Island and Lizard Island, western side of Blue Lagoon, Lizard Island (14 $\left.41^{\circ} \mathrm{S} 145^{\circ} 27^{\prime} \mathrm{E}\right), 2-3 \mathrm{~m}$, coral rubble, 7 October 2001, coll. J.M. Guerra-García \& S.J. Keable (QLD 1469); 8 specimens (4 0 and 4 우), AM P.61715, Coconut Beach, Lizard Island $\left(14^{\circ} 41^{\prime} \mathrm{S} 145^{\circ} 27^{\prime} \mathrm{E}\right), 6 \mathrm{~m}$, fine live hard coral encrusted with calcareous red algae and filamentous red/green/brown algal turf, 8 October 2001, coll. J.M. Guerra-García \& S.J. Keable (QLD 1476); 3 specimens (1 9 and 2 juveniles), AM P.61716, Crystal Beach, Lizard Island (14²41'S $\left.145^{\circ} 27^{\prime} \mathrm{E}\right)$, $5 \mathrm{~m}$, thick branching coral rubble encrusted with red algal turf and black sponge half buried in sediment, 9 October 2001, coll. J.M. Guerra-García \& S.J. Keable (QLD 1483); 1 juvenile, AM P.61717, Crystal Beach, Lizard Island $\left(14^{\circ} 41^{\prime} \mathrm{S} 145^{\circ} 27^{\prime} \mathrm{E}\right), 4 \mathrm{~m}$, thin branching live coral heavily encrusted 


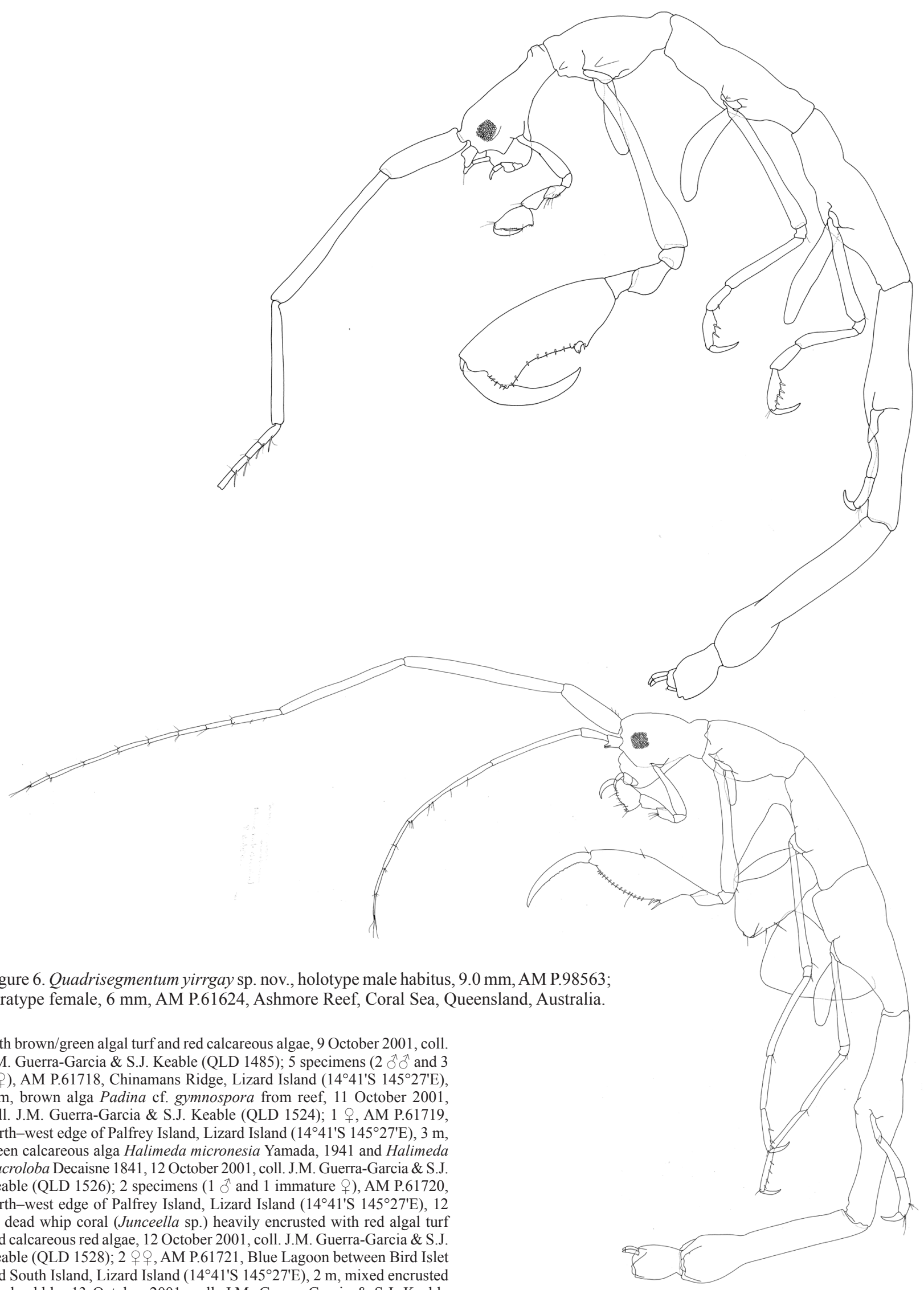
(15. 13 October 2001, coll. J.M. Guerra-Garcia \& S.J. Keable (QLD 1536); 1 , AM P.61722, reef slope on south side of South Island, Lizard Island (14'41'S $\left.145^{\circ} 27^{\prime} \mathrm{E}\right), 20 \mathrm{~m}$, gorgonian (cf. Paracis sp.), 14 October 2001, coll. J.M. Guerra-Garcia \& S.J. Keable (QLD 1541); many specimens, AM P.61723, reef slope on south side of South Island, Lizard 

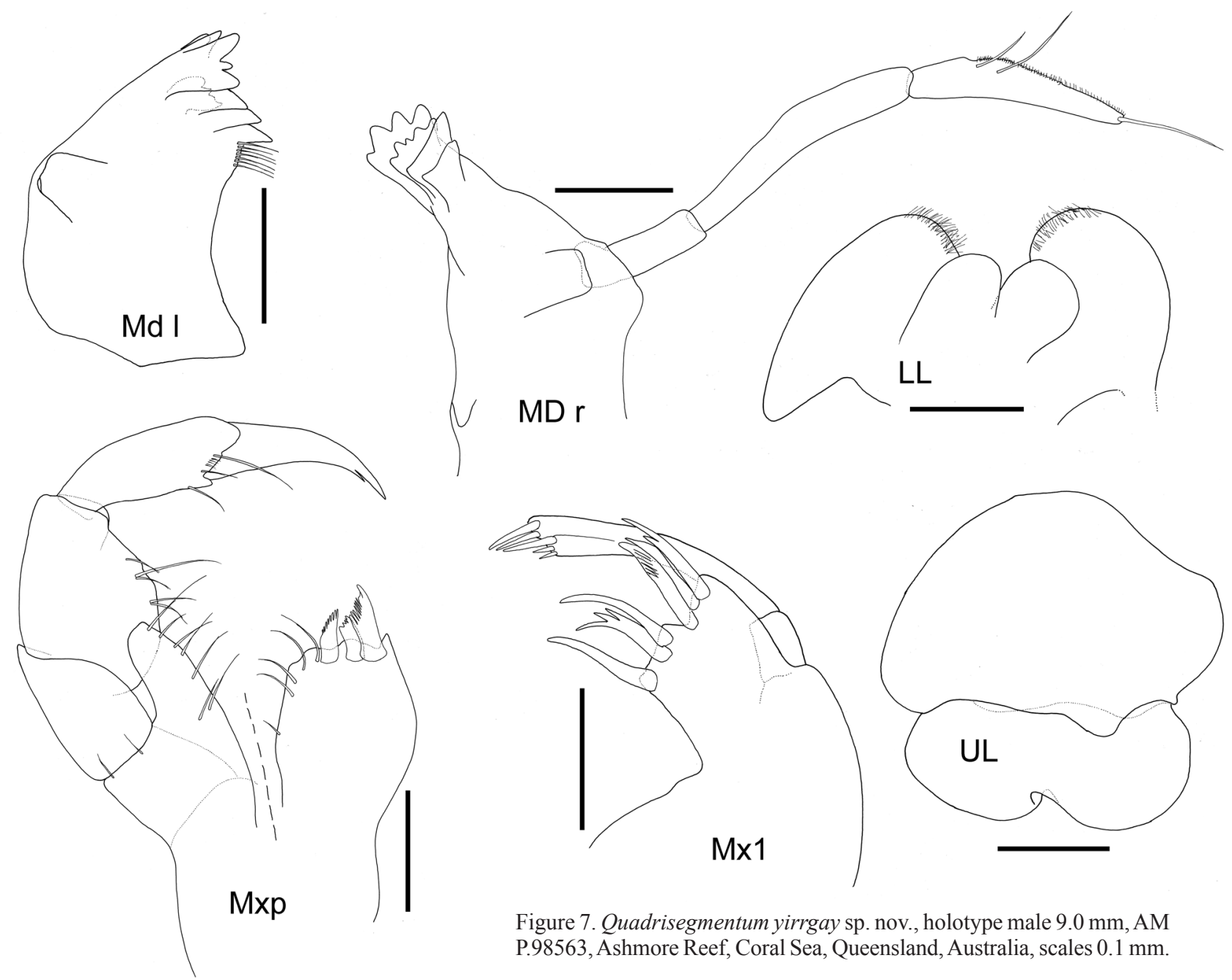

Figure 7. Quadrisegmentum yirrgay sp. nov., holotype male $9.0 \mathrm{~mm}, \mathrm{AM}$ P.98563, Ashmore Reef, Coral Sea, Queensland, Australia, scales $0.1 \mathrm{~mm}$.

Island ( $\left.14^{\circ} 41^{\prime} \mathrm{S} 145^{\circ} 27^{\prime} \mathrm{E}\right), 25 \mathrm{~m}$, gorgonian (cf. Menella sp.), 14 October 2001, coll. J.M. Guerra-Garcia \& S.J. Keable (QLD 1544); 1 ô, AM P.61724, reef slope on south side of South Island, Lizard Island (14\% $41^{\prime} \mathrm{S}$ $145^{\circ} 27^{\prime} \mathrm{E}$ ), $20 \mathrm{~m}$, gorgonian (cf. Mopsella sp.), 14 October 2001, coll. J.M. Guerra-Garcia \& S.J. Keable (QLD 1545); 2 ô ô, AM P.61725, north-east side of Lizard Island, reef slope south of "Washing Machine" (14²4'S $\left.145^{\circ} 27^{\prime} \mathrm{E}\right), 18 \mathrm{~m}$, brown sponge cf. Clathria reinwardti, 16 October 2001, coll. J.M. Guerra-Garcia \& S.J. Keable (QLD 1565); 1 , AM P.61726, north-east side of Lizard Island, reef slope south of "Washing Machine" $\left(14^{\circ} 41^{\prime} \mathrm{S} 145^{\circ} 27^{\prime} \mathrm{E}\right), 6 \mathrm{~m}$, green calcareous alga Halimeda macroloba, 16 October 2001, coll. J.M. Guerra-Garcia \& S.J. Keable (QLD 1568); 1 ㅇ, AM P.61727, north-east side of Lizard Island, reef slope south of "Washing Machine" (14⒋ $\left.11^{\prime} \mathrm{S} 145^{\circ} 27^{\prime} \mathrm{E}\right), 5 \mathrm{~m}$, sediment with green alga Halimeda cylindracea Decaisne 1842, Udotea and Caulerpa spp., 16 October 2001, coll. J.M. Guerra-Garcia \& S.J. Keable (QLD 1569); 2 specimens (1 $\delta$ and 1 juvenile), AM P.61728, north-east side of Lizard Island, reef slope south

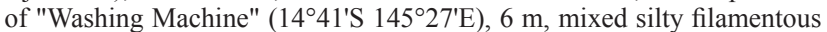
red algae and green alga Caulerpa taxifolia (M.Vahl) C.Agardh 1817, 16 October 2001, coll. J.M. Guerra-Garcia \& S.J. Keable (QLD 1573); 1 , AM P.61729, Pidgin Point, Lizard Island (14ㄴ1'S $\left.145^{\circ} 27^{\prime} \mathrm{E}\right), 20 \mathrm{~m}$, sponge cf. Clathria reinwardti, 18 October 2001, coll. J.M. Guerra-Garcia \& S.J. Keable (QLD 1581); 1 Oे, AM P.61730, reef just south-west of Osprey Island, Lizard Island $\left(14^{\circ} 41^{\prime} \mathrm{S} 145^{\circ} 27^{\prime} \mathrm{E}\right), 10-12 \mathrm{~m}$, fine-medium sand with some shell, 18 October 2001, coll. J.M. Guerra-Garcia \& S.J. Keable (QLD 1588); 1 mature + , AM P.61731, Granite Bluff, Lizard Island (14² $40^{\prime} \mathrm{S}$

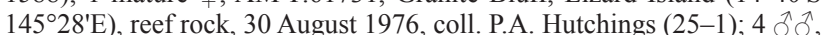
AM P.61732, northern end of Mermaid Beach, Lizard Island (14\%41'S $\left.145^{\circ} 27^{\prime} \mathrm{E}\right), 4 \mathrm{~m}$, brown alga Padina sp., 12 October 2001, coll. J.M. GuerraGarcia \& S.J. Keable (QLD 1533).

Tasman Sea: 7 specimens (6 $\delta \hat{\delta}$ and 1 ㅇ), AM P.61581, patch reef in lagoon, Middleton Reef, Elizabeth and Middleton Reefs (29 $27^{\prime} 36^{\prime \prime S}$ $159^{\circ} 06^{\prime} 42^{\prime \prime E}$ ), prolific coral cover on top of bommie, 6 December 1987
(Site 12); 1 , AM P.61582, The Sound, large alcove near lagoon entrance, Middleton Reef, Elizabeth and Middleton Reefs (29 27'42"S 159 05'24"E), rubble wash, 5 December 1987 (Site 7); 1 3. AM P.61475, reef front south of North Passage, straight out from Signal Point, Lord Howe Island $\left(31^{\circ} 31^{\prime} 36^{\prime \prime} \mathrm{S} 159^{\circ} 03^{\prime} \mathrm{E}\right)$ coralline algae, coll. G.D. Fenwick, 11 May 1977 (LHA 17).

Papua New Guinea: o $6.1 \mathrm{~mm}$, whole animal SEM pin mount, AM P.97779, The Quarry, near the village of Bunu 2, Madang ( $4^{\circ} 46^{\prime} 30^{\prime \prime} \mathrm{S}$ $145^{\circ} 48^{\prime} \mathrm{E}$ ), coral slope with rubble, sand, ascidian Didemnum molle (Herdmann, 1886) and sponges, 21 January 1990, coll. J.K. Lowry (PNG 69); ㅇ $6.1 \mathrm{~mm}$, AM P.98565, The Quarry, near the village of Bunu 2, Madang $\left(4^{\circ} 46^{\prime} 30^{\prime \prime} \mathrm{S} 145^{\circ} 48^{\prime} \mathrm{E}\right)$, coral slope with rubble, sand, ascidian Didemnum molle and sponges, 21 January 1990, coll. J.K. Lowry (PNG 69); 3 specimens ( $2 \sigma^{\lambda}$ and 1 mature 9 ), AM P.61534, base of reef slope, outer face, Horseshoe Reef, Bootless Inlet ( $\left.9^{\circ} 30^{\prime} 30^{\prime \prime} \mathrm{S} 147^{\circ} 15^{\prime} 30^{\prime \prime} \mathrm{E}\right)$ green calcareous alga Halimeda opuntia (Linnaeus) J.V. Lamouroux 1816, 28 October 1980, coll. S.P. Arnam \& J.K. Lowry (PNG-23); 1 mature 9 , AM P.61533, Horseshoe Reef, Bootless Inlet ( $9^{\circ} 30^{\prime} 30^{\prime \prime} S$ 147 $\left.17^{\circ} 10^{\prime \prime} \mathrm{E}\right)$ coral rubble from reef front slope, 29 October 1980, coll. S.P. Arnam \& J.K. Lowry (PNG-32); 1 , AM P.61536, Horseshoe Reef, Port Moresby $\left(9^{\circ} 36^{\prime} 30^{\prime \prime S} 147^{\circ} 17^{\prime} 54 " \mathrm{E}\right)$ gorgonian, 2 November 1980, coll. A.J. Bruce (AJB 206); 4 specimens (3 0 and 1 damaged juvenile), AM P.61537, Lion Island, Manunouma Islands (9 $\left.32^{\circ} 17^{\prime \prime S} 147^{\circ} 06^{\prime} 13 " \mathrm{E}\right)$, gorgonian, 5 November 1980, coll. A.J. Bruce (AJB-208); 4 specimens (3 ôे ô and 1 mature P), AM P.61535, outside Horseshoe Reef, Bootless Inlet $\left(9^{\circ} 30^{\prime} 30^{\prime \prime} \mathrm{S}\right.$ $147^{\circ} 17^{\prime} 30^{\prime \prime E}$ ), crinoids from outer reef face, 9 November 1980 , coll. S.P. Arnam \& J.K. Lowry (PNG-63); 74 specimens, AM P.61531, The Quarry, near the village of Bunu $2\left(4^{\circ} 46^{\prime} 30^{\prime \prime} \mathrm{S} 145^{\circ} 48^{\prime} \mathrm{E}\right)$, coral slope with rubble, sand, ascidian Didemnum molle and sponges, 21 January 1990, coll. J.K. Lowry (PNG 69); 1 , AM P.61532, The Quarry, near the village of Bunu $2\left(4^{\circ} 46^{\prime} 30^{\prime \prime} \mathrm{S} 145^{\circ} 48^{\prime} \mathrm{E}\right)$, coral slope with rubble, sand and black sponge, coll. J.K. Lowry, 21 January 1990 (PNG 68). 


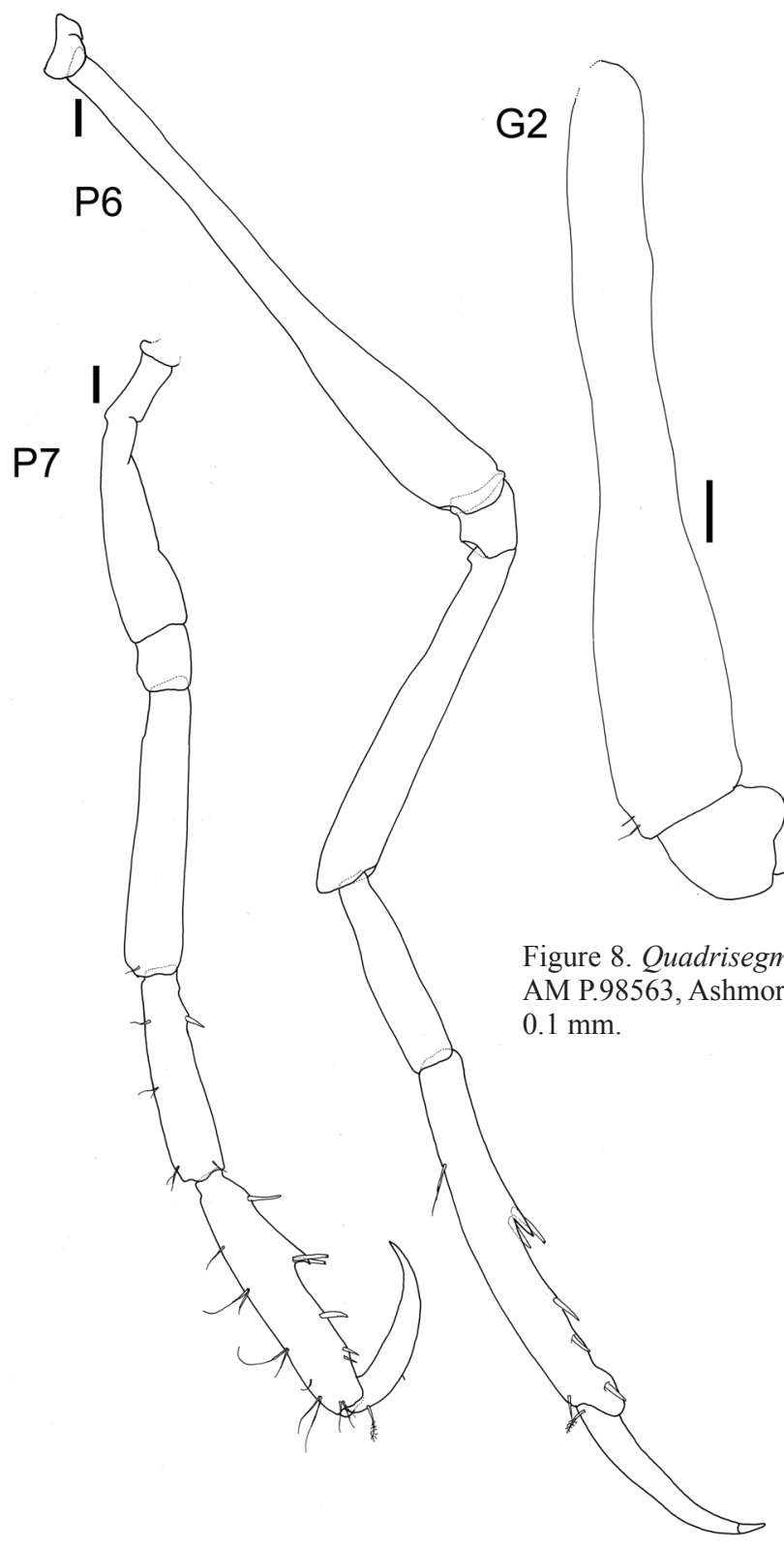

Type locality. Ashmore Reef, off Great Barrier Reef, Coral Sea, Queensland, Australia $\left(10^{\circ} 26^{\prime} 16^{\prime \prime} \mathrm{S} 144^{\circ} 25^{\prime} 47^{\prime \prime} \mathrm{E}\right)$. (The present "Ashmore Reef" is the different "Ashmore Reef", Timor Sea, north-west Western Australia, type locality for Q. triangulum)

Etymology. "Yirrgay" is the lost spoken language of a coastal indigenous group in Far North Queensland. Applied as a noun in apposition.

Description. (Based on holotype $\widehat{\partial}$, body length $9.0 \mathrm{~mm}, \mathrm{AM}$ P.98563 and male, body length, $6.1 \mathrm{~mm}$, SEM AM P.97779 for abdomen).

Body Somites sub-slender. Head and pereonite 1 combined, completely fused, suture absent, with pair of apically truncate dorsodistal projections. Pereonite 2, with small acute anterolateral projection and larger acute mid lateral projection. Pereonite 3, with minute mid-lateral projection. Pereonites 4-6 without anterior or mid-lateral projections
Antenna 1 more than $1 / 3 \times$ body length (broken); peduncle article 2 longest, $2 \times$ article 1 ; article 3 length $0.8 \times$ article 2 ; flagellum with more than 4 articles (broken).

Upper lip weakly notched. Mandible right, incisor with 4 teeth, lacinia mobilis with 5 teeth followed by 2 plates; palp 3-articulate; article 2 longest; article 3 with 1 distal and 2 midlateral slender setae, anterodistal margin pubescent. Mandible left, incisor with 5 teeth, lacinia mobilis followed by 2 plates; Lower lip setose on outer lobe. Maxilla 1 outer plate with 6 robust setae; palp 2-articulate, article 2 with 3 long and 2 short robust seate. Maxilliped basal endite (inner plate) larger than ischial endite (outer plate), basal endite with 3 apically serrate robust setae; ischial endite (outer plate) margin with several slender setae; palp 4-articulate; article 2 lateral expanded, with 5 setae; article 3 with sub-triangular distal projection.

Pereon. Gnathopod 1 basis similar in length to ischium, merus and carpus combined; carpus subequal to merus length, posterior margin with slender setae; propodus triangular; palm defined by prominent, curved, bifid process with 2 apical robust setae; dactylus falcate, inner margin lined with setules. Gill 2 subovate, length $0.6 \times$ pereonite 2 length. Gnathopod 2 begins $1 / 3$ along posterior margin of pereonite 2 ; basis length $1.4 \times$ pereonite 2 length, $2.1 \times$ ischium, merus and carpus combined; merus subrectangular, distally swollen (length $2 \times$ width), longer than to carpus; carpus subrectangular (length $2 \times$ width); propodus subovate, longer than wide (length $2.1 \times$ width); palm $60 \%$ of posterior margin, 3 proximal projections each with robust seta.

Gill 3 elongate, length $0.6 \times$ pereonite 3 . Pereopod 3 basis longest, $0.4 \times$ pereopod 3 length; ischium $0.1 \times$ basis length; merus $0.7 \times$ basis length; carpus $0.25 \times$ basis length; propodus $1.1 \times$ carpus length with 2 large robust setae along palm, dactylus recurved. Gill 4 subequal to gill 3. Pereopod 


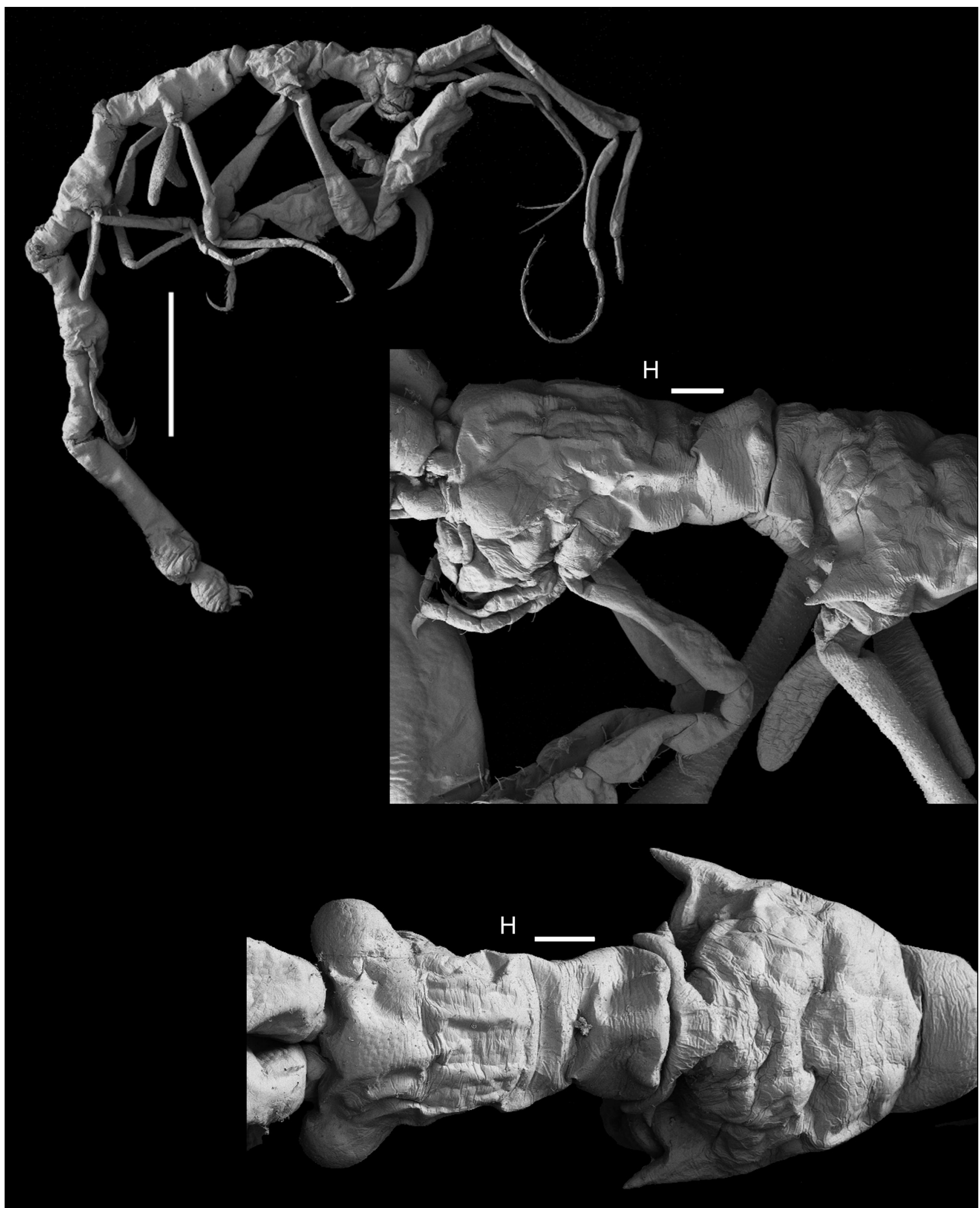

Figure 9. Quadrisegmentum yirrgay sp. nov., male, $6.1 \mathrm{~mm}$, AM P.97779, Madang, Papua New Guinea, scales habitus $1 \mathrm{~mm}$, all other scales $100 \mu \mathrm{m}$.

4 shorter than pereopod 3 , length $0.30 \times$ body; basis longest, $0.6 \times$ pereopod 3 length; ischium $0.25 \times$ basis length; merus $0.6 \times$ basis length; carpus $0.3 \times$ basis length; propodus longer than carpus, length $1.1 \times$ carpus with 4 large robust setae along palm; dactylus recurved.
Pereopod 5 article 1 length $0.9 \times$ article 2 ; article 2 longest, $1.1 \times$ article 3 ; dactylus recurved. Pereopod 6 basis longest, $0.37 \times$ pereopod 6 length; ischium $0.08 \times$ basis length; merus $0.6 \times$ basis length; carpus $0.33 \times$ basis length, anterior margin without robust setae; propodus $0.6 \times$ basis length, anterior 


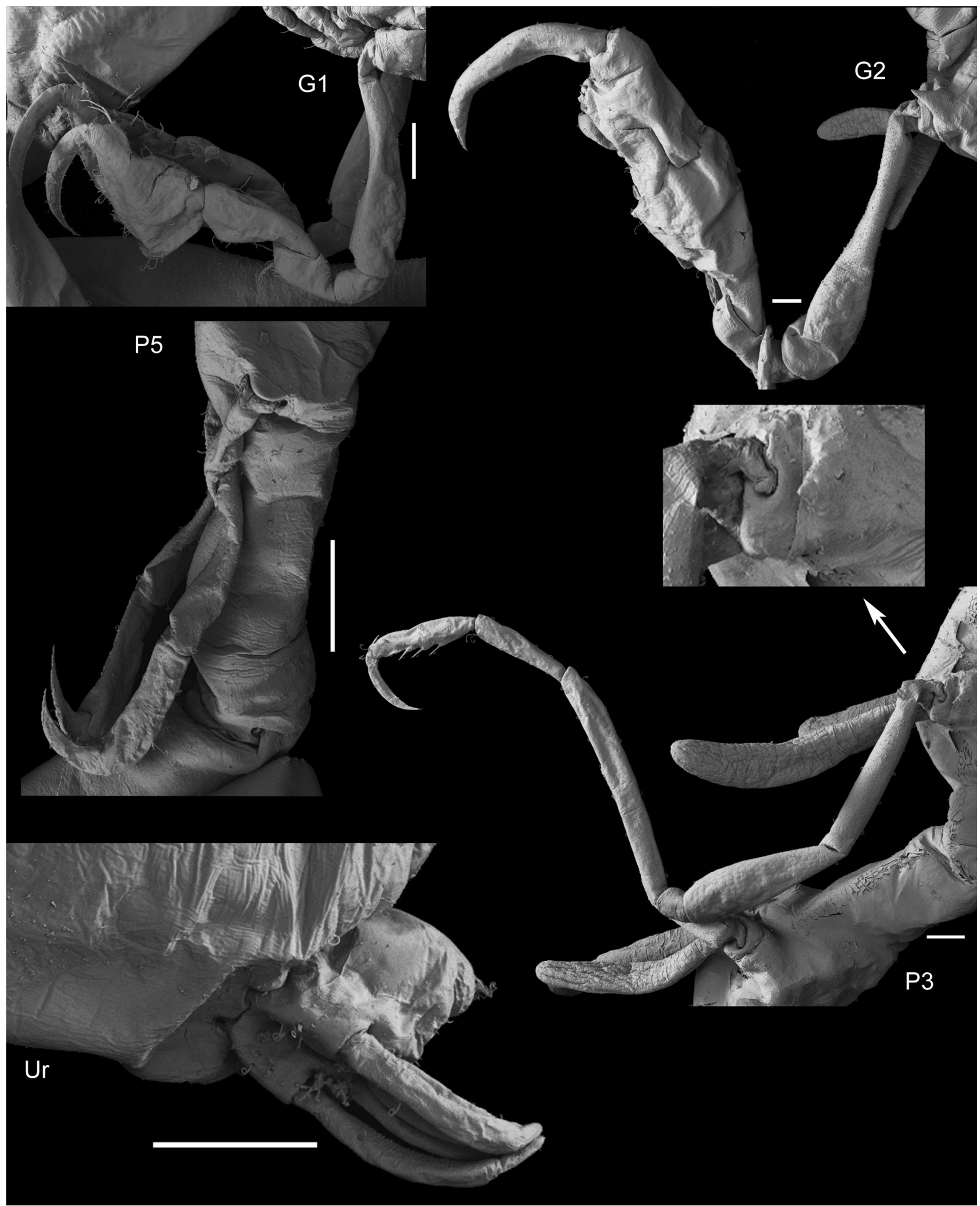

Figure 10. Quadrisegmentum yirrgay sp. nov., male, $6.1 \mathrm{~mm}$, AM P.97779, Madang, Papua New Guinea, scales Ur $20 \mu \mathrm{m}$, all other scales $100 \mu \mathrm{m}$.

margin with 4 groups of long robust setae (proximal group only with paired robust setae); dactylus recurved. Pereopod $70.8 \times$ pereopod 6 ; basis $0.27 \times$ pereopod 7 length; ischium $0.25 \times$ basis length; merus length subequal to basis; carpus $0.65 \times$ basis length, anterior margin with 1 robust seta; propodus $0.8 \times$ basis length, anterior margin with 3 groups of long robust setae (middle group only with paired robust setae) and distal small robust setae; dactylus recurved.

Pleon. Uropod 1 elongate, without setae (broken). Uropod 2 (broken). Telson small, with a pair of setae (Based on SEM AM P.97779). 


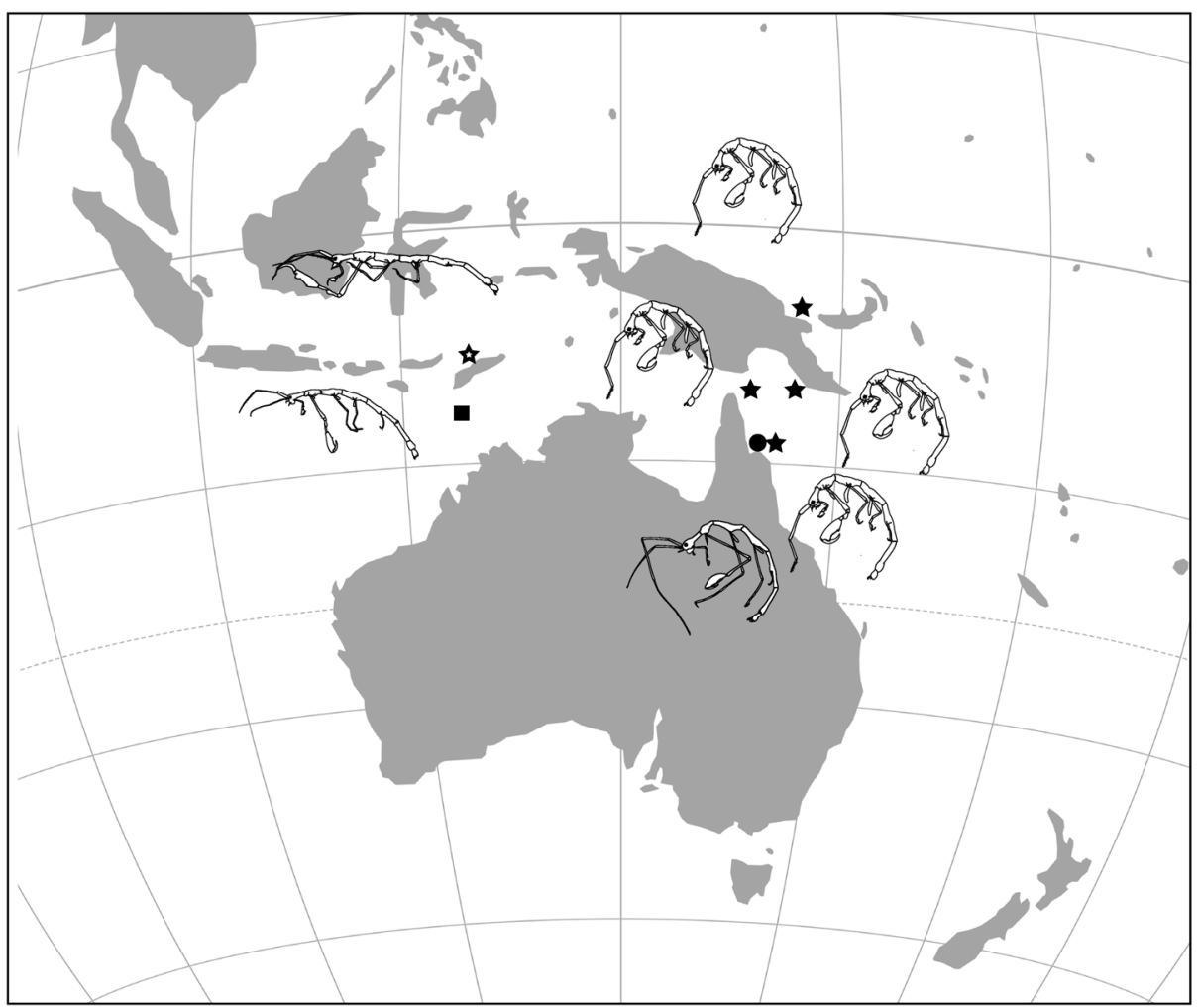

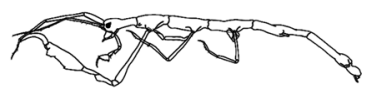

Q. atauro sp. nov

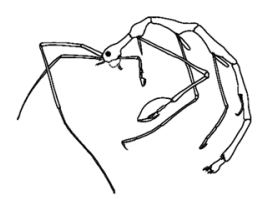

- Q. lowryi Guerra-García, 2006

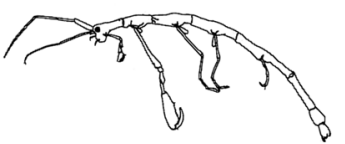

Q. triangulum Hirayama, 1998

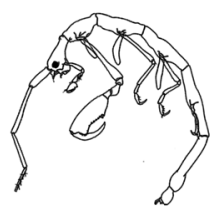

Q. yirrgay sp. nov

Figure 11. Distribution of world species of Quadrisegmentum Hirayama, 1988.

Female. (based on paratype AM P.61624). Pereonite 2 with small anterolateral and small acute mid-lateral projections. Pereonite 4 with rounded anterolateral projection. Antenna 1 long $0.75 \times$ body length; article 2 longest, $2 \times$ article 1 length; flagellum with 8 articles. Antenna 2 shorter than antenna 1; flagellum 4-articulated, length $1 / 2 \times$ antenna 1 .

Remarks. See remarks for $Q$. atauro sp. nov.
Distribution. Australia: Ashmore Reef, Coral Sea, Lizard Island (current study; Guerra-Garcia, 2006; Guerra-Garcia \& Lowry, 2009). Lord Howe Island. (Guerra-García, 2006). Elizabeth and Middleton Reef(Guerra-García, 2006). Papua New Guinea: Motupore Island, Manunouma Islands, Port Morseby and Madang (Guerra-García, 2003).

\section{Key to species of Quadrisegmentum Hirayama, 1988}

1 Gnathopod 2 merus extremely elongate, more than 5 times carpus length (in males and females) Q. lowryi

Gnathopod 2 merus not elongate, similar or up to twice carpus length (in males and females)

2 Pereonite 2 without dorsal projections; pereonites 1-3 gills short, subovate

Pereonite 2 with anterolateral and/or mid-lateral projections; pereonites $1-3$ gills elongate, rectilinear

3 Male gnathopod 2 carpus shorter than merus; pereopod 6 carpus without robust setae (in males) Q. yirrgay sp. nov.

Male gnathopod 2 carpus subequal to merus length; pereopod 6 carpus with 2 robust setae (in males) 
ACKNOWLEDGMENTS. The Australian Museum field work in TimorLeste was made possible by a gracious donation to the Australian Museum Foundation. We thank Helen Stoddart, AM, for assistance with collections and Sue Horner, NTM, for loan of type material.

\section{References}

Agardh, C. A. 1817. Synopsis algarum Scandinaviae, adjecta dispositione universali algarum. pp. i-xl, 1-135. Lundae [Lund]: Ex officina Berlingiana.

Decaisne, J. 1841. Plantes de l'Arabie Heureuse, recueillies par M. P.-E. Botta et décrites par M. J. Decaisne. Archives du Muséum d'Histoire Naturelle, Paris 2: 89-199, pls V-VII.

Decaisne, J. 1842. Mémoire sur les corallines ou polypiers calcifères (la seconde partie du «Essais sur une classification des algues et des polypiers calcifères de Lamourous». Annales des Sciences Naturelles, Botanique, Seconde Série 18: 96-128.

Guerra-García, J. M. 2003. Caprellids (Crustacea: Amphipoda) from Papua New Guinea, with the description of a new species. Helgoland Marine Research 57: 100-109. http://dx.doi.org/10.1007/s10152-003-0144-4

Guerra-García, J. M. 2006. Caprellidae (Crustacea: Amphipoda) from the Great Barrier Reef and adjacent localities. Records of the Australian Museum 58(3): 417-458. http://dx.doi.org/10.3853/j.0067-1975.58.2006.1451

Guerra-García, J. M., and J. K. Lowry, 2009. Caprellidae. In Benthic Amphipoda (Crustacea: Peracarida) of the Great Barrier Reef, ed. J. K. Lowry and A. A. Myers. Zootaxa 2260: 290-327.

Herdman, W. A. 1886. Report on the Tunicata collected during the voyage of H.M.S. Challenger during the years 1873-1876. Pt II, Ascidiae compositae. Report of the Scientific Results of the Voyage of H.M.S. Challenger 1873-1876, Zool. 14(38), pp. 1-425.

Hirayama, A. 1988. A ghost shrimp with four-articulate fifth pereopods (Crustacea: Caprellidea: Phtisicidae) from northwest Australia. Zoological Science 5: 1089-1093.

Hughes, L. E. 2015. Ampithoidae and Maeridae amphipods from Timor-Leste (Crustacea: Peracarida). Records of the Australian Museum 67(3): 83-108. http://dx.doi.org/10.3853/j.2201-4349.67.2015.1644

Krapp-schickel, T., and J. M. Guerra-García. 2005. Littoral Caprellidae (Crustacea: Amphipoda) from Indonesia, with the description of a new species. Bollettino del Museo Civico di Storia Naturale di Verona, 29, 2005 Botanica Zoologia: 47-62.

Lamouroux, J. V. F. 1816. Histoire des polypiers coralligènes flexibles, vulgairement nommés zoophytes. pp. i-lxxxiv, chart, 1-560, [560, err], pls I-XIX, uncol. by author. Caen: De l'imprimerie de F. Poisson.
Lim, J. H. C., B. A. R. Azman, and I. Takeuchi. 2012. Microtripus tinggiensis, new genus and species (Amphipoda: Caprellidea: Phtisicidae) from Pulau Tinggi, East Johor Islands Archipelago, Malaysia. Proceedings of the Biological Society of Washington 125 (3): 241-251.

http://dx.doi.org/10.2988/11-40.1

Linnaeus, C. 1758. Systema naturae per regna tria naturae: secundum classes, ordines, genera, species, cum characteribus, differentiis, synonymis, locis (in Latin) 10th edition. Stockholm: Laurentius Salvius; 1758.

Mayer, P. 1903. Die Caprellidae der Siboga-Expedition. SibogaExpeditie, Monographie 34: 1-160.

McCain, J. C., and J. E. Steinberg. 1970. Amphipoda I. Caprellidea I. Fam. Caprellidae. Crustaceorum Catalogus 2: 1-78.

Scinto, A., G. Bavestrello, M. Boyer, M. Previati, and C. Cerrano. 2008. Gorgonian mortality related to a massive attack by caprellids in the Bunaken Marine Park (North Sulawesi, Indonesia). Journal of the Marine Biological Association of the United Kingdom 88(4): 723-727.

http://dx.doi.org/10.1017/S002531540800129x

Takeuchi, I. 1993. Is the Caprellidea a monophyletic group? Journal of Natural History, 27: 947-964.

http://dx.doi.org/10.1080/00222939300770581

Takeuchi, I. 2015. A new species of Paraproto (Crustacea: Amphipoda: Phtisicidae) collected from the South Shetland Islands, Antarctica. Polar Science 9: 368-373.

http://dx.doi.org/10.1016/j.polar.2015.08.002

Takeuchi, I., and J. K. Lowry. 2016. A taxonomic study on the Phtisicidae (Crustacea: Amphipoda) of New South Wales, Australia. Journal of Natural History 50: 603-648. http://dx.doi.org/10.1080/00222933.2015.1079338

Vassilenko, S. V. 1968. [On the taxonomy and basic development lines of the family Caprellidae (Amphipoda, Caprellidea)]. Akademiya Nauk SSSR, Doklady 183: 1461-1464.

Veron, J. E. N., L. M. Devantier, E. Turak, A. L. Green, S. Kininmonth, M. Stafford-Smith, and N. Peterson. 2009. Delineating the coral triangle. Galaxea, Journal of Coral Reef Studies 11: 91-100. http://dx.doi.org/10.3755/galaxea.11.91

Yamada, Y. 1941. [On the species of Halimeda from Micronesia]. Kagaku Nanyo 4: 108-121, 15 figs.

Manuscript submitted 30 March 2016, revised 13 September 2016, and accepted 7 October 2016. 